\title{
Breakdown of Fermi-liquid theory in a cuprate superconductor
}

\author{
R.W. Hill, Cyril Proust ${ }^{\dagger}$ and Louis Taillefer \\ Canadian Institute for Advanced Research and \\ Department of Physics, University of Toronto, Toronto, Ontario M5S 1A7, Canada \\ P. Fournier $\ddagger$ and R.L. Greene \\ Center for Superconductivity Research \\ Department of Physics, University of Maryland, \\ College Park, Maryland 20742, USA
}

(Dated: December 13, 2001)

\begin{abstract}
The behaviour of electrons in solids is remarkably well described by Landau's Fermi-liquid theory, which says that even though electrons in a metal interact they can still be treated as well-defined fermions, called "quasiparticles". At low temperature, the ability of quasiparticles to transport heat is strictly given by their ability to transport charge, via a universal relation known as the Wiedemann-Franz law, which no material in nature has been known to violate. High-temperature superconductors have long been thought to fall outside the realm of Fermi-liquid theory, as suggested by several anomalous properties, but this has yet to be shown conclusively. Here we report on the first experimental test of the Wiedemann-Franz law in a cuprate superconductor, $(\mathrm{Pr}, \mathrm{Ce})_{2} \mathrm{CuO}_{4}$. Our study reveals a clear departure from the universal law and provides compelling evidence for the breakdown of Fermi-liquid theory in high-temperature superconductors.
\end{abstract}

PACS numbers: $74.70 . T x, 74.25 . F y$

\section{INTRODUCTION}

Landau's Fermi-liquid theory is the definitive theory of electrons in metals [1], or more generally fermions in condensed matter, and a major landmark of 20th century physics. For example, it is the necessary foundation for the theory of superconductivity by Bardeen, Cooper and Schrieffer (BCS) [2]. In essence, it says that even in the presence of interactions the low-energy excitations of a system of mobile fermions can still be described in terms of well-defined fermionic particles, called "quasiparticles", with charge $e$, spin $\frac{1}{2}$ and a mass $m^{\star}$, the latter being renormalized by interactions. The WiedemannFranz (WF) law is one of the basic properties of a Fermi liquid, reflecting the fact that the ability of a quasiparticle to transport heat is the same as its ability to transport charge, provided it cannot lose energy through collisions. Reported as an empirical observation by Wiedemann and Franz in 1853 [3], the law states that the heat conductivity $\kappa$ and the electrical conductivity $\sigma$ of a metal are related by a universal constant:

$$
\frac{\kappa}{\sigma T}=\frac{\pi^{2}}{3}\left(\frac{k_{B}}{e}\right)^{2} \equiv L_{0}
$$

where $T$ is the absolute temperature, $k_{B}$ is Boltzmann's constant and $L_{0}=2.45 \times 10^{-8} \mathrm{~W} \Omega \mathrm{K}^{-2}$ is Sommerfeld's value for the Lorenz ratio $L \equiv \kappa / \sigma T$ [ [1].

The linear power of temperature in Eq. (1) comes from the linear temperature dependence of the fermionic specific heat, through the relation between heat transport and heat capacity. In kinetic theory,

$$
\kappa=\frac{1}{3} c v l
$$

where $c$ is the specific heat of the carriers, $v$ is their average velocity and $l$ their mean free path. Provided the mean free path between collisions is independent of temperature, as it is for the strictly elastic processes that operate at $T \rightarrow 0$, the heat conduction has the same $T$ dependence as the specific heat. So for electrons, it is also linear in $T$, and given by Eq. (1). By contrast, bosonlike excitations such as phonons or magnons give rise to a low-temperature specific heat and a heat conductivity as $T \rightarrow 0$ which are proportional to $T^{3}$.

Theoretically, electrons are predicted to obey the WF law at $T \rightarrow 0$ in a very wide range of environments [5]: in both three dimensions or two dimensions (but not strictly in one dimension), for any strength of disorder and interaction (in the scaling theory of disorder and interaction) [6], scattering [7] and magnetic field [8]. Experimentally, the WF law does appear to be universal at $T \rightarrow 0$ : no material has been reported to violate it. (Note that a departure from Eq. (1) has in fact been reported for silver [9, but is in contradiction with other measurements 10, 11, including our own [12].) This is true not only of simple metals like copper [10, 11], but also of systems with strong electron correlations - such as the heavy fermion compounds $\mathrm{CeAl}_{3}$ [13], $\mathrm{CeCu}_{6}$ [14] and $\mathrm{UPt}_{3}$ |15] - or highly anisotropic conduction - such as the quasi-2D system $\mathrm{Sr}_{2} \mathrm{RuO}_{4}$ [16] (a ruthenate which is isostructural to the cuprate $\mathrm{La}_{2} \mathrm{CuO}_{4}$ ), the quasi-1D organic conductor (TMTSF) ${ }_{2} \mathrm{ClO}_{4}$ 17, or the 2D electron gas in a MOSFET [18]. Even in the presence of so-called non-Fermi liquid behaviour, where the electrical resistivity is seen to deviate from the standard $T^{2}$ dependence, the WF law still holds at $T \rightarrow 0$, as observed in $\mathrm{CeNi}_{2} \mathrm{Ge}_{2}$ [19]. All this strongly suggests that the ground state of every 
metal investigated thus far is a Fermi liquid. Of course, if the metallic state gives way to a superconducting state at low temperature, then the WF law is entirely violated, as the charge is no longer transported by fermions but by Cooper pairs, which carry no entropy.

In 1986, high-temperature superconductivity was discovered in a class of oxides called cuprates, and fifteen years hence the fundamental question about these materials still remains: are they host to a new state of matter? In particular, are they Fermi liquids in their ground state, once superconductivity is removed (either by application of a magnetic field or by doping)? Anderson proposed early on 20] that the cuprates are indeed fundamentally different from other metals in that they are Mott insulators. When a small number of electrons or holes are doped into the $\mathrm{CuO}_{2}$ planes of their crystal structure, these materials can conduct charge reasonably well and also form a superconducting state. It is believed that the basic excitations of a doped Mott insulator are not fermionic quasiparticles as in other metals - with charge, spin and heat all carried by one and the same particle. Instead, the electron is thought to "fractionalize" into a neutral spin- $\frac{1}{2}$ fermion called a "spinon" and a spinless charge-e boson called a "holon", or a "chargon" 21, a phenomenon called "spin-charge" separation.

Even though this bold proposal for a fundamentally new state of matter continues to be at the heart of several current theories of the cuprates 22, there has been little experimental evidence for such spin-charge separation. On the contrary, for example, a BCS/Fermiliquid theory of $d$-wave quasiparticles appears to work rather well in the superconducting state of hole-doped cuprates, at least at low energy and near optimal doping [23. Conceptually, a simple way to investigate the possibility of spin-charge separation is to measure the properties of charge transport and compare them to those of either spin or entropy transport. In this Article, we report on the first test of this kind, where both the charge conductivity $\sigma$ and the heat conductivity $\kappa$ of a cuprate superconductor are measured in the normal state at $T \rightarrow 0$. We observe a good, metallic-like nearly temperature-independent charge transport at low temperature without the corresponding heat transport expected from the WF law. The normalized Lorenz number $L / L_{0}$ goes from being much less than unity at $T \rightarrow 0$ to being greater than unity above $0.2 \mathrm{~K}$. This is highly suggestive of spin-charge separation and a compelling demonstration that the ground state of cuprates can fall outside the realm of Fermi-liquid theory.

\section{THE MATERIAL}

The cuprate material used for this study is $\mathrm{Pr}_{2-x} \mathrm{Ce}_{x} \mathrm{CuO}_{4-y}$ (PCCO), with a doping concentration of $x=0.15$, which is roughly the optimum value for maximizing the critical temperature for super- conductivity, where $T_{c} \simeq 20 \mathrm{~K}$. The addition of $x$ Ce atoms on $\mathrm{Pr}$ sites adds $x$ electrons to the $\mathrm{CuO}_{2}$ planes of the parent insulating compound, the Mott insulator $\mathrm{Pr}_{2} \mathrm{CuO}_{4}$. PCCO is the electron-doped analog of the hole-doped material $\mathrm{La}_{2-x} \mathrm{Sr}_{x} \mathrm{CuO}_{4-y}$ (LSCO), wherein the addition of $x$ Sr atoms on La sites removes $x$ electrons from (adds $x$ holes to) the $\mathrm{CuO}_{2}$ planes. In LSCO, the optimum doping is also at approximately $x=0.15$, with a maximum $T_{c} \simeq 40 \mathrm{~K}$. For reasons that are not clear, both materials have critical temperatures that are significantly lower than those of several other cuprates, such as $\mathrm{YBa}_{2} \mathrm{Cu}_{3} \mathrm{O}_{7-\delta} \quad(\mathrm{Y}-123), \mathrm{Bi}_{2} \mathrm{Sr}_{2} \mathrm{CaCu}_{2} \mathrm{O}_{8+\delta}$ (Bi-2212) and $\mathrm{Tl}_{2} \mathrm{Ba}_{2} \mathrm{CuO}_{6+\delta}$ (Tl-2201), which all have a maximal $T_{c}$ around $90 \mathrm{~K}$. Nevertheless, all cuprates share the same basic crystal structure, namely that of a stack of $\mathrm{CuO}_{2}$ planes, and the same generic dependence on doping. The technical advantage of LSCO and PCCO is that the magnetic field needed to destroy superconductivity is correspondingly lower, with upper critical field values of $\simeq 50 \mathrm{~T}$ and $\simeq 10 \mathrm{~T}$, respectively. Because these fields can be achieved in the laboratory, it is possible to investigate their lowtemperature transport properties in the normal state, such as was done for charge conductivity by Boebinger et al. on LSCO [24 and by Fournier et al. on PCCO [25].

\section{CRYSTALS}

The single crystal of PCCO used in this study was grown with a flux technique described elsewhere 26, 27, using a $\mathrm{Al}_{2} \mathrm{O}_{3}$ crucible and an oxygen reduction treatment at $1000{ }^{\circ} \mathrm{C}$. The concentration of cerium was fixed at $x=0.15$. Contacts were made with silver epoxy, diffused at $500{ }^{\circ} \mathrm{C}$ for 1 hour, and were used to measure both electrical resistivity and thermal conductivity. The electrical resistance of contacts was typically $\simeq 1 \Omega$. The crystal was in the shape of a platelet, $37 \mu \mathrm{m}$ thick and $720 \mu \mathrm{m}$ wide, with $1.0 \mathrm{~mm}$ separation between voltage contacts. The superconducting transition temperature $T_{c}$ was obtained from three different measurements: as the end of the drop in resistivity, the end of the diamagnetic drop in susceptibility and the onset of a small peak in thermal conductivity. In all cases, $T_{c}=20 \mathrm{~K}$ to within less than $\pm 0.5 \mathrm{~K}$. The width of the transition is $6 \mathrm{~K}$ in resistivity, $3 \mathrm{~K}$ in magnetisation and about $1 \mathrm{~K}$ in thermal conductivity. The broad resistive transition, seen both as a function of temperature and magnetic field, is most likely due to an inhomogeneous distribution of oxygen near the surface. The narrower transition obtained in thermal conductivity suggests that this inhomogeneity is confined to a negligible volume of the sample, and therefore does not affect bulk measurements such as heat transport. Note that all results presented in this paper were reproduced on several other single crystals grown in similar conditions, with comparable $T_{c}$ and resistivity. 


\section{CHARGE TRANSPORT}

The temperature dependence of the electrical resistivity is shown in Fig. 1 for different magnetic fields applied parallel to the $c$-axis of the tetragonal crystal structure. The current is made to flow in the $\mathrm{CuO}_{2}$ planes, where the conduction is some $10^{4}$ times better than perpendicular to the planes (at room temperature). Above $25 \mathrm{~K}$, the temperature dependence is not linear as in most optimally-doped cuprates, but follows a power law $a+b T^{\alpha}$ with $\alpha \simeq 1.7$, and has a magnitude characteristic of a reasonably good metal: $a \simeq 25 \mu \Omega \mathrm{cm}$ and $\rho(300 \mathrm{~K}) \simeq 140 \mu \Omega \mathrm{cm}$. The effect of a magnetic field is to rapidly suppress the superconducting transition initially, but then more slowly at high fields, giving rise to a curve $T_{c}(H)$ that has strong positive curvature. From the magnetoresistance at $0.3 \mathrm{~K}$ shown in the lower inset, one can see that the entire sample has completed its transition to the normal state by $13.5 \mathrm{~T}$. Note, however, that the bulk of the sample is no longer superconducting above $8 \mathrm{~T}$, the field beyond which the low-temperature heat transport ceases to evolve (see Fig. 2 below).

The overall behaviour of the resistivity in PCCO is similar to that of hole-doped cuprates when overdoped.

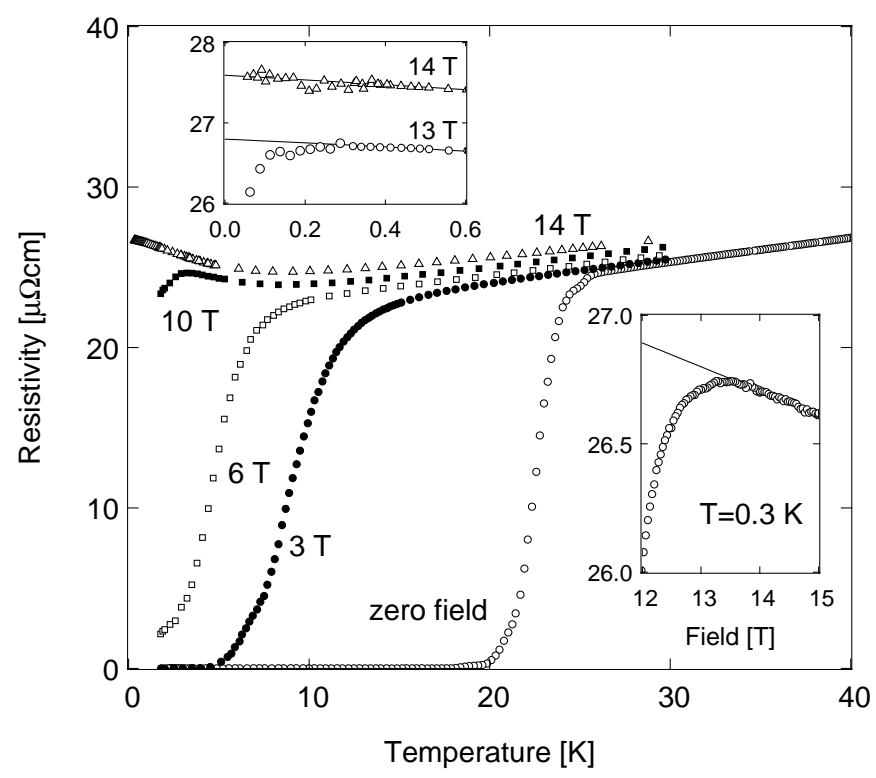

FIG. 1: Electrical resistivity of PCCO vs temperature for a current in the basal plane at different values of magnetic field applied normal to the plane. Upper inset: zoom of low temperature dependence at $13 \mathrm{~T}$ and $14 \mathrm{~T}$, the latter being shifted upwards by $0.8 \mu \Omega \mathrm{cm}$ for clarity. Lines are fits to the data below $1.0 \mathrm{~K}$. Lower inset: Resistivity vs magnetic field at $T=0.3 \mathrm{~K}$, showing that the crystal has fully completed its transition to the normal state by $13.5 \mathrm{~T}$. The line is a linear fit to the negative magnetoresistance beetween 13.5 and $15 \mathrm{~T}$.
Indeed, when doped to have a $T_{c}$ of $15 \mathrm{~K}$, Tl-2201 has a resistivity which goes as $T^{1.75}$ with values of $10 \mu \Omega \mathrm{cm}$ at low temperature and $180 \mu \Omega \mathrm{cm}$ at room temperature, and a resistive critical field with a strong upward curvature reaching a value of $16 \mathrm{~T}$ at $T \rightarrow 0$ [28]. One difference is the slight upturn seen at low temperature in PCCO and not in Tl-2201. The resistivity in the normal state (at $14 \mathrm{~T}$ ) increases by about $10 \%$ in going from $8 \mathrm{~K}$ down to $0.3 \mathrm{~K}$ (see Fig. 1). Similar but much larger upturns were observed in $\mathrm{Nd}_{2-x} \mathrm{Ce}_{x} \mathrm{CuO}_{4}$ (NCCO) [29, 30] and PCCO [30] at lower carrier concentrations, and attributed to weak localization. In support of this interpretation is the suppression of the upturn by a transverse magnetic field, also observed in our samples (see inset of Fig. 1). This negative magnetoresistance eventually flattens to a constant beyond $25 \mathrm{~T}$, equal to $25.2 \mu \Omega \mathrm{cm}$ at $0.5 \mathrm{~K} 31$.

In summary, once the superconducting order has been completely suppressed throughout the sample, namely in a magnetic field larger than $13.5 \mathrm{~T}$, the charge transport of this cuprate material at low temperature is that of a fairly good quasi-two-dimensional metal, with a residual resistivity $\rho(T \rightarrow 0)=\rho_{0}=26.8 \mu \Omega \mathrm{cm}$ in $14 \mathrm{~T}$, which corresponds to a conductivity per $\mathrm{CuO}_{2}$ plane of $60 e^{2} / h$ (i.e. $\left.k_{F} l \simeq 60\right)$.

\section{HEAT TRANSPORT}

Fifteen years after the discovery of high-temperature superconductivity, very little is known about the normal state of cuprates at low temperature. The ability to suppress superconductivity in optimally-doped PCCO with a field of only $14 \mathrm{~T}$ provides a rare opportunity to shed light on the fundamental nature of the electron system in these materials. In the present study, this was done by measuring the transport of heat as $T \rightarrow 0$. The thermal conductivity $\kappa(T)$ of PCCO is shown in Fig. 2 at temperatures below $0.3 \mathrm{~K}$, for heat flowing parallel to the $\mathrm{CuO}_{2}$ planes. The data is plotted as $\kappa / T$ vs $T^{2}$ in order to easily separate the contributions of phonons and electrons, using the fact that phonons are expected to have a heat conductivity which varies as $T^{3}$ when their mean free path reaches the size of the crystal at sufficiently low temperature. A straight line extrapolating to zero in $\kappa / T$ vs $T^{2}$ is indeed the asymptotic $(T \rightarrow 0)$ behaviour observed in insulators [32], including the Mott insulator $\mathrm{YBa}_{2} \mathrm{Cu}_{3} \mathrm{O}_{6}$ (i.e. Y-123 without any doped carriers) [33]. This is also the behaviour of conventional ( $s$-wave) superconductors. The absence of a residual linear term $\kappa_{0} / T$, defined as the value of $\kappa / T$ as $T \rightarrow 0$, means that there are no mobile fermionic excitations at low temperature. In insulators, this is due to the lack of electronic carriers, while in superconductors it is because electrons have all formed Cooper pairs at $T<<T_{c}$. The heat is then conducted entirely by phonons. In contrast, a residual linear term is observed when fermionic excitations are present, 


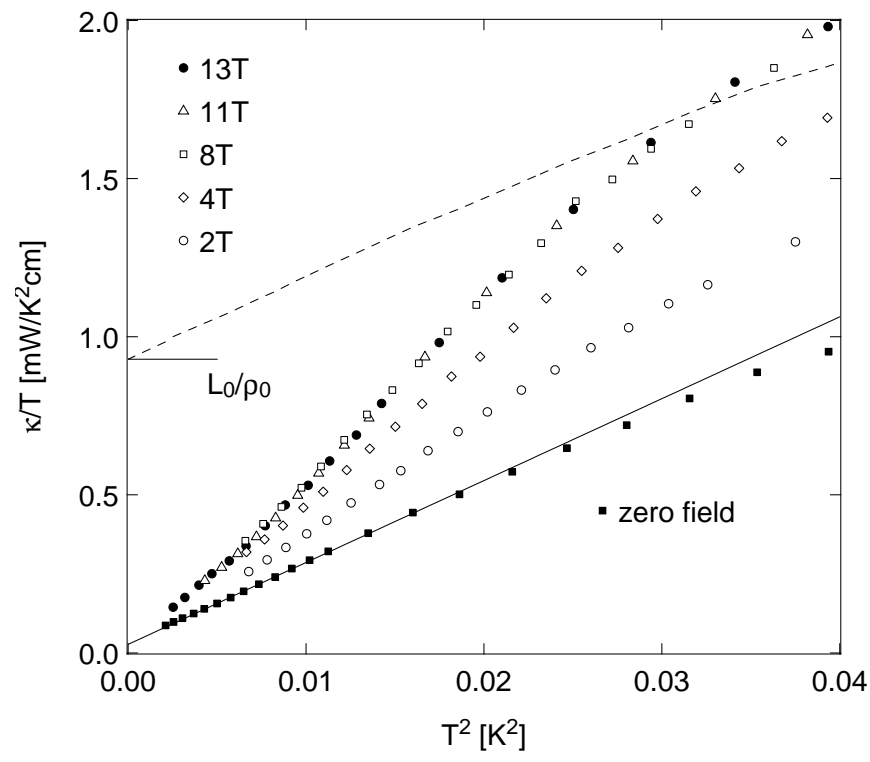

FIG. 2: Thermal conductivity of PCCO for a heat current in the basal plane, plotted as $\kappa / T$ vs $T^{2}$, at different values of the magnetic field applied normal to the plane. The solid line is a linear fit to the zero-field data below $130 \mathrm{mK}$. The dashed line shows the behaviour of a Fermi-liquid with the residual resistivity $\rho_{0}$ of this sample, calculated as the sum of a constant electronic linear term via Eq. (1) and a phonon conductivity given by the zero-field data.

as in the following three instances: 1) in metals, with a residual linear term given precisely by the WF law; 2) in the vortex state of superconductors, where a magnetic field induces a residual linear term by generating delocalized quasiparticles [34]; 3) in $d$-wave superconductors, where zero-energy quasiparticles are induced by impurity scattering [35.

\section{Superconducting state}

In the absence of a magnetic field, there is only a very small residual linear term in the thermal conductivity of PCCO. From data on four different crystals, we find $\kappa_{0} / T=0.03-0.07 \mathrm{~mW} \mathrm{~K}^{-2} \mathrm{~cm}^{-1}$. This is considerably smaller than the residual linear term observed in three hole-doped cuprates, at optimal doping: $\kappa_{0} / T=0.14$, 0.15 and $0.11 \mathrm{~mW} \mathrm{~K}^{-2} \mathrm{~cm}^{-1}$, in Y-123 33, 36], Bi-2212 [37, 38] and LSCO [39], respectively. Within BCS theory applied to a $d$-wave superconductor, this fermionic residual heat conduction is expected, arising from zeroenergy quasiparticles induced by impurity scattering near the nodes in the $d_{x^{2}-y^{2}}$ gap function [35]. In the case of Bi-2212, the excellent quantitative agreement between theory and experiment has been viewed as a strong validation of a Fermi-liquid quasiparticle picture for the superconducting state [23, 37], at least at optimal doping and low energies.
Several tentative explanations may be offered for the weakness of the residual linear term in PCCO. The first possibility is the localization of $d$-wave quasiparticles, investigated by several authors 40]. It was invoked recently as a possible cause for the absence of a residual linear term in $\mathrm{YBa}_{2} \mathrm{Cu}_{4} \mathrm{O}_{8}$ (Y-124) [4]. However, it is not clear why localization should occur in Y-124 and not in Y-123, given that these two cuprates have comparable structure and properties. It seems more plausible in PCCO, because of the highly two-dimensional nature of its conduction - some 100 times more anisotropic than either Y-124 or LSCO. On the other hand, Bi-2212 is equally anisotropic, yet shows delocalized quasiparticles. A second scenario is the absence of nodes in the gap structure of PCCO. This would eliminate any residual linear term (at least in zero magnetic field). The symmetry of the order parameter need not be $s$-wave, but could have the form $d+i x$, where $x$ is a subdominant component of either $s$ or $d_{x y}$ symmetry. The possibility of a $T=0$ transition in the order parameter from $d$ to $d+i x$ as a function of doping has been raised in the context of a possible quantum critical point in the phase diagram of cuprates [42]. For PCCO, however, this would seem to be in conflict with evidence for a rather pure $d_{x^{2}-y^{2}}$ symmetry as determined in a recent tricrystal experiment [43]. The other difficulty is that, as we shall see below, a magnetic field normal to the $\mathrm{CuO}_{2}$ planes never induces a residual linear term, implying that the minimum gap would have to be a sizable fraction of the maximum gap (i.e. $x$ comparable to $d$ ). This would have to be reconciled with the penetration depth data 44, 45]. A third possibility, proposed recently by Granath et al. [46], is the removal of the nodes as a result of static stripe ordering.

\section{Normal state}

As seen in Fig. 2, the basic effect of a magnetic field is to rapidly increase the thermal conductivity of PCCO (at finite temperature). Given that the phonon conductivity cannot exceed its value at zero field (where the phonon mean free path has reached its maximum value), this increase must be due to excitations of electronic origin. The field-induced conduction (over and above the zero-field curve) grows rapidly (and approximately linearly) with field at low fields to eventually saturate at high fields, where $\kappa$ is absolutely constant above 8 $\mathrm{T}$. This is compelling evidence that the thermodynamic upper critical field in this sample is at most $8 \mathrm{~T}$ and the bulk of the crystal is in the normal state beyond that. Indeed, in all known superconductors, whether $s$ wave or $d$-wave, the electronic thermal conductivity is always affected by the magnetic field when in the vortex state, and seen to saturate above $H_{c 2}$ (see for example Ref. 477). Such an $H_{c 2}$ agrees reasonably well with the criterion of zero resistance, achieved at about 


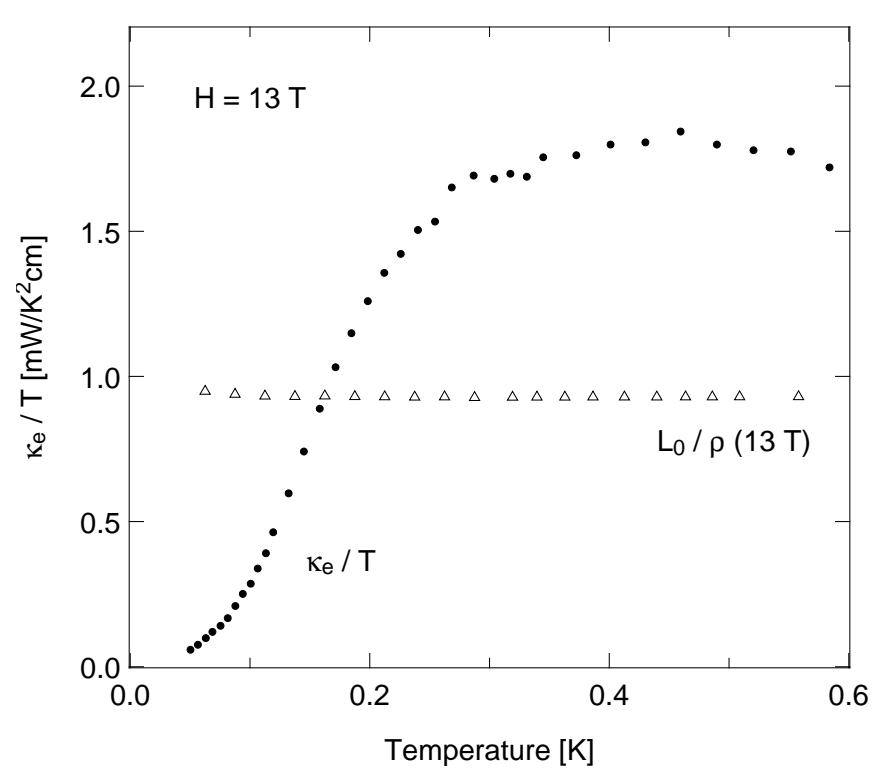

FIG. 3: Comparison of charge conductivity $\sigma(T)=1 / \rho(T)$, plotted as $L_{0} / \rho(T)$ (open triangles), and electronic heat conductivity $\kappa_{e}$, plotted as $\kappa_{e} / T$ (full circles), as a function of temperature in the normal state at $H=13 \mathrm{~T}$. The electronic contribution to the heat conduction is the difference between the measured $\kappa(13 \mathrm{~T})$ and the phonon contribution $\kappa_{p h}(13$ $\mathrm{T})$, estimated in the text. In a Fermi liquid, the curve of $\kappa_{e} / T$ would lie precisely on top of the data for $L_{0} / \rho$. Below $0.15 \mathrm{~K}, \kappa_{e} \sim T^{3.6}$

$6 \mathrm{~T}$, and also agrees with the $H_{c 2}$ values reported previously in thin films of PCCO [25, 48] and NCCO 48, 49] at $x=0.15$. A roughly linear growth of $\kappa$ with field is also seen, for example, in the borocarbide superconductor $\mathrm{LuNi}_{2} \mathrm{~B}_{2} \mathrm{C}$ [34], however with a major difference: in $\mathrm{LuNi}_{2} \mathrm{~B}_{2} \mathrm{C}$, the field induces a linear term at $T \rightarrow 0$. Furthermore, this finite residual linear term ultimately reaches a value in the normal state given precisely by the WF law. In striking contrast, a magnetic field, even as high as $13 \mathrm{~T}$, almost twice $H_{c 2}$, never induces a residual linear term in PCCO. Given the known value of the electrical resistivity at $T \rightarrow 0$ and $13 \mathrm{~T}$, namely $\rho_{0}=26.8 \mu \Omega \mathrm{cm}$, the WF law predicts a residual linear term $\kappa_{0} / T=L_{0} / \rho_{0}=0.91 \mathrm{~mW} \mathrm{~K}^{-2} \mathrm{~cm}^{-1}$, as drawn in Fig. 2. (Note that weak localization is not expected to alter the WF law appreciably [6, as verified experimentally in layered graphene [50].)

This complete violation of the WF law shows that the non-superconducting ground state of this cuprate material is not a Fermi liquid. The charge carriers responsible for the good electrical conduction do not display the expected fermionic heat transport.

It is instructive to consider the effect of increasing the temperature above zero. Contributions to the heat transport from the electron system $\left(\kappa_{e}\right)$ and from the ionic lattice $\left(\kappa_{p h}\right)$ will add to give the measured thermal con- ductivity: $\kappa(T)=\kappa_{e}(T)+\kappa_{p h}(T)$. These two systems are coupled. It is unambiguous that phonons are scattered by electrons since the crystal conducts heat better at zero field than at $13 \mathrm{~T}$ beyond $0.6 \mathrm{~K}$. In other words, as the temperature is raised, inelastic scattering processes become increasingly effective at limiting the phonon heat flow, and we expect the dominant process to be electronphonon scattering.

Given this observed electron-phonon coupling, the total phonon scattering in field is the sum of whatever scatters phonons in zero field plus the added scattering that comes from the field-induced electronic excitations. The thermal resistivity of phonons due to electron scattering is given by $W_{e}^{p h}=B T^{-2}$ [51], where the constant $B$ depends on the strength of the electron-phonon coupling, the electronic density of states, and various other factors. This means that the phonon conductivity at low $T$ is given by $\kappa_{p h}=\kappa(0)\left[1+\kappa(0) W_{e}^{p h}\right]^{-1}$, where $\kappa(0)$ is the measured zero-field conductivity, assumed to be essentially all phononic. We can put a reasonable lower bound on the electron-phonon coupling parameter $B$ by requiring that the resulting $\kappa_{e} / T$ does not decrease with $T$. Adjusting $B$ such that $\kappa_{e} / T$ is constant above $0.3 \mathrm{~K}$ gives the curve shown in Fig. 3 for $H=13 \mathrm{~T}$ (full circles). The same can be done for other fields with the resulting values of $\kappa_{e} / T=0.6,1.2$ and $1.9 \mathrm{~mW} \mathrm{~K}^{-2} \mathrm{~cm}^{-1}$ and corresponding values of $B=0.22,0.4$, and 0.68 $\mathrm{K}^{3} \mathrm{~m} \mathrm{~W}^{-1}$ for $H=2,4$, and 8 (or 13) $\mathrm{T}$, respectively. (In comparison, electron-phonon coupling in copper gives $B=4-8 \mathrm{~K}^{3} \mathrm{~m} \mathrm{~W}^{-1}[51]$.) Note the self-consistency of this analysis whereby $B$ scales with the value of $\kappa_{e} / T$ (above $0.3 \mathrm{~K}$ ); in other words, by increasing the field thereby increasing the density of electronic excitations one increases both the electronic heat conduction and the electron scattering of phonons.

From Fig. 3, we see that $\kappa_{e}(T) / T$ exceeds the value of $L_{0} / \rho$ appropriate for a Fermi liquid. In other words, the low-energy excitations of the electron system violate the WF law not only at $T \rightarrow 0$, where $\kappa_{e}(T) / T \ll L_{0} / \rho_{0}$, but also at finite temperatures, where $\kappa_{e}(T) / T>L_{0} / \rho$ (above $0.2 \mathrm{~K}$ ). It is important to appreciate that qualitatively the latter violation is independent of our choice of $B$, in the sense that even if $B$ is set to zero, $\kappa_{e} / T$ still exceeds $L_{0} / \rho$, in this case by $30 \%$ at $0.25 \mathrm{~K}$. We stress that $B=0$ is unphysical, as it would lead to a negative $\kappa_{e} / T$ for $T>0.6 \mathrm{~K}$. It is also independent of our assumption that $\kappa(0)$ is entirely phononic; indeed if some part of $\kappa(0)$ is in fact electronic, then the violation turns out to be even more pronounced. In other words, while there is no accurate way to extract the electronic contribution at $13 \mathrm{~T}$, the fact that the WF law is violated is independent of our model for the phonon conduction and the electronphonon scattering. The result $\kappa_{e}(T) / T \simeq 2 L_{0} / \rho_{0}$ above $0.3 \mathrm{~K}$ is obtained in several crystals, even though $\rho\left(T_{c}\right)$ varies in the range 20 to $40 \mu \Omega \mathrm{cm}$.

We have investigated the possibility that our mea- 
surement gradually ceases to detect the electronic heat current as electrons fall out of thermal equilibrium with the phonon bath when the temperature approaches $T=0$. One can imagine this happening when the electrical contact resistance between the heater and the sample is too large and the heat is predominantly carried by the phonons. This might then explain the drop in $\kappa_{e} / T$ measured below $0.3 \mathrm{~K}$ or so (see Fig. 3). Two facts lead us to rule out electron-phonon decoupling as the main mechanism for the drop. First, identical data is obtained by sending heat directly through the electron system using photons (as in Ref. [52). Secondly, a similar drop is observed in samples of optimally-doped $(\mathrm{La}, \mathrm{Sr})_{2} \mathrm{CuO}_{4}$, where we have achieved electrical contact resistances that are 100 times lower than in our PCCO samples, namely $10 \mathrm{~m} \Omega$. We therefore believe this low-temperature drop is intrinsic and not specific to PCCO.

\section{CONCLUSION}

The cuprate PCCO is the first material to violate the Wiedemann-Franz law. The breakdown of this robust signature of Fermi-liquid theory suggests that the fundamental entities that carry heat, charge (and spin) in the cuprates are not the usual Landau quasiparticles. The fact that the electron system conducts heat in a way which is largely unrelated to the way it carries charge at low temperature points to the existence of neutral excitations responsible for much of $\kappa_{e}$. If these neutral excitations are fermions, i.e. spinons, they should contribute a constant term to $\kappa_{e} / T$, as may well be the case for $T>0.3 \mathrm{~K}$. The drop seen at the very lowest temperatures then remains to be explained, and the possibility of spinon localization should be considered.

The present study is currently limited to a single carrier concentration, near optimal doping, in the whole phase diagram. It will be interesting to investigate the evolution with doping of this emerging picture. If one speculates that the WF law is satisfied in overdoped cuprates, where Fermi-liquid theory is generally assumed to hold, our results on optimally-doped PCCO suggest that a violation of the law is a property of underdoped cuprates. A comprehensive study will shed light on the gradual breakdown of Fermi-liquid theory in the cuprates as the carrier concentration is reduced towards the Mott insulating state.

\section{Acknowledgements}

We thank C. Lupien, E. Boaknin, M. Sutherland, M. Chiao, R. Gagnon, J. Brooks, L. Balicas and B. Brandt for their invaluable help in various aspects of the measurements. This work was supported by the Canadian Institute for Advanced Research and funded by NSERC of Canada. LT ackowledges the support of a Premier's Research Excellence Award from the Government of Ontario. The work in Maryland was supported by the NSF Division of Condensed Matter Physics.

Correspondence should be addressed to L.T. (Louis.Taillefer@utoronto.ca).

$\dagger$ Present address: Laboratoire National des Champs Magnétiques Pulsés, 143 Avenue de Rangueil, BP 4245, 31432 Toulouse, Cedex 04, France.

¥ Present address: Département de Physique, Université de Sherbrooke, Sherbrooke, Québec J1K 2R1, Canada.

[1] Landau, L.D. The theory of a Fermi liquid. Soviet Physics JETP 3, 920-925 (1957).

[2] Bardeen, J., Cooper, L.N. \& Schrieffer, J.R. Theory of superconductivity. Phys. Rev. 108, 1175-1204 (1957).

[3] Wiedemann G. \& Franz R. Annl. Phys. 89, 497-532 (1853).

[4] Ashcroft, N.W. \& Mermin, N.D. Solid State Physics (HRW, Philadelphia, 1976), p. 322.

[5] Kearney, M.J. \& Butcher, P.N. Thermal transport in disordered systems. J. Phys. C: Solid State Phys. 21, L265L270 (1988).

[6] Castellani, C., DiCastro, C., Kotliar, G., Lee, P.A. \& Strinati, G. Thermal conductivity in disordered interacting-electron systems. Phys. Rev. Lett. 59, 477480 (1987).

[7] Chester, G.V. \& Tellung, A. The law of Wiedemann and Franz. Proc. Phys. Soc. 77, 1005-1013 (1961).

[8] Smrcka, L. \& Streda, P. Transport coefficients in strong magnetic fields. J. Phys. C: Solid State Phys. 10, 21532161 (1977).

[9] Gloos, K., Mitschka, C., Pobell, F. \& Smeibidl, P. Thermal conductivity of normal and superconducting metals. Cryogenics 30, 14-18 (1990).

[10] Anderson, A.C., Peterson, R.E. \& Robichaux, J.E. Thermal and electrical conductivity of $\mathrm{Ag}$ and $\mathrm{Pt}$ below $1 \mathrm{~K}$. Phys. Rev. Lett. 20, 459-461 (1968).

[11] Rumbo, E.R. Transport properties of very pure copper and silver below 8.5 K. J. Phys. F: Metal Phys. 6, 85-98 (1976).

[12] Hill, R.W. \& Boaknin, E. Personal communication.

[13] Ott, H.R., Marti, O. \& Hulliger, F. Low temperature thermal conductivity of $\mathrm{CeAl}_{3}$. Solid State Commun. 49, 1129-1131 (1984).

[14] Amato, A. et al. Transport properties of $\mathrm{CeCu}_{6}$ at very low temperature. J. Magn. Magn. Mater. 63 \& 64, 300302 (1987).

[15] Suderow, H. et al. Thermal conductivity and gap structure of the superconducting phases of $\mathrm{UPt}_{3}$. J. Low Temp. Phys. 108, 11-30 (1997).

[16] Tanatar, M.A. et al. Thermal conductivity of superconducting $\mathrm{Sr}_{2} \mathrm{RuO}_{4}$ in oriented magnetic fields. Phys. Rev. B. 63, 064505-1-7 (2001).

[17] Belin, S. \& Behnia, K. Thermal conductivity of superconducting (TMTSF $)_{2} \mathrm{ClO}_{4}$ : evidence for a nodeless gap. Phys. Rev. Lett. 79, 2125-2128 (1999). 
[18] Syme, R.T., Kelly, M.J. \& Pepper, M. Direct measurement of the thermal conductivity of a two-dimensional electron gas. J. Phys.: Condens. Matter 1, 3375-3380 (1989).

[19] Kambe, S. et al. Spin-fluctuation mediated thermal conductivity around the magnetic instability of $\mathrm{CeNi}_{2} \mathrm{Ge}_{2}$. J. Low Temp. Phys. 117, 101-112 (1999).

[20] Anderson, P.W. The resonating valence bond state in $\mathrm{La}_{2} \mathrm{CuO}_{4}$ and superconductivity. Science 235, 1196-1198 (1987).

[21] Kivelson, S.A., Rokhsar, D.S. \& Sethna, J.P. Topology of the resonating valence bond state: Solitons and high- $\mathrm{T}_{c}$ superconductivity. Phys. Rev. B 35, 8865-8868 (1987).

[22] Senthil, T. \& Fisher, M.P.A. $\mathrm{Z}_{2}$ gauge theory of electron fractionalization in strongly correlated systems. Phys. Rev. B 62, 7850-7881 (2000); and references therein.

[23] Orenstein, J. \& Millis, A.J. Advances in the physics of high-temperature superconductivity. Science 288, 468474 (2000).

[24] Boebinger, G.S. et al. Insulator-to-metal crossover in the normal state of $\mathrm{La}_{2-x} \mathrm{Sr}_{x} \mathrm{CuO}_{4}$ near optimum doping. Phys. Rev. Lett. 77, 5417-5420 (1996).

[25] Fournier, P. et al. Insulator-metal crossover near optimal doping in $\mathrm{Pr}_{2-x} \mathrm{Ce}_{x} \mathrm{CuO}_{4}$ : Anomalous normal-state low temperature resistivity. Phys. Rev. Lett. 81, 4720-4723 (1998).

[26] Peng, J.L., Li, Z.Y. \& Greene, R.L. Growth and characterization of high-quality single crystals of $\mathrm{R}_{2-x} \mathrm{Ce}_{x} \mathrm{CuO}_{4-y} \quad(\mathrm{R}=\mathrm{Nd}, \mathrm{Sm})$. Physica $C$ 177, $79-85$ (1991).

[27] Brinkmann, M. et al. Crystal growth of high- $T_{c}$ superconductors $\operatorname{Pr}_{2-x} \mathrm{Ce}_{x} \mathrm{CuO}_{4+\delta}$ with substitutions of $\mathrm{Ni}$ and Co for Cu. J. Crystal Growth 163, 369-376 (1996).

[28] Mackenzie, A.P. et al. Resistive upper critical field of $\mathrm{Tl}_{2} \mathrm{Ba}_{2} \mathrm{CuO}_{6}$ at low temperatures and high magnetic fields. Phys. Rev. Lett. 71, 1238-1241 (1993).

[29] Harus, G.I et al. Two-dimensional weak localization effects in high-temperature superconductor $\mathrm{Nd}_{2-x} \mathrm{Ce}_{x} \mathrm{CuO}_{4-\delta}$. Soviet JETP 116, 1-12 (1999).

[30] Fournier, P. et al. Anomalous saturation of the phase coherence length in underdoped $\mathrm{Pr}_{2-x} \mathrm{Ce}_{x} \mathrm{CuO}_{4}$ thin films. Physical Review B 62, R11993-R11996 (2000).

[31] Hill, R.W. et al. Personal communication.

[32] Thacher, P.D. Effect of boundaries and isotopes on the thermal conductivity of LiF. Phys. Rev. 156, 975-988 (1967).

[33] Taillefer, L. et al. Universal heat conduction in $\mathrm{YBa}_{2} \mathrm{Cu}_{3} \mathrm{O}_{6.9}$. Phys. Rev. Lett. 79, 483-486 (1997).

[34] Boaknin, E. et al. Highly anisotropic gap function in borocarbide superconductor $\mathrm{LuNi}_{2} \mathrm{~B}_{2}$ C. Phys. Rev. Lett. accepted Nov 2001.

[35] Durst, A.C. \& Lee, P.A. Impurity-induced quasiparticle transport and universal-limit Wiedemann-Franz violation in $d$-wave superconductors. Phys. Rev. B 62, 1270-
1290 (2000).

[36] Chiao, M. et al. Quasiparticle transport in the vortex state of $\mathrm{YBa}_{2} \mathrm{Cu}_{3} \mathrm{O}_{6.9}$. Phys. Rev. Lett. 82, 2943-2946 (1999).

[37] Chiao, M. et al. Low-energy quasiparticles in cuprate superconductors: A quantitative analysis. Phys. Rev. B 62, 3554-3558 (2000).

[38] Behnia, K. et al. Features of heat conduction in organic and cuprate superconductors. J. Low Temp. Phys. 117, 1089-1098 (1999).

[39] Taillefer, L. \& Hill, R.W. Heat transport in hightemperature superconductors. Physics in Canada, 56, 237-240 (2000).

[40] Senthil, T. et al. Quasiparticle transport and localization in high- $T_{c}$ superconductors. Phys. Rev. Lett. 81, 47044707 (1998); and references therein.

[41] Hussey, N.E. et al. Absence of residual quasiparticle conductivity in the underdoped cuprate $\mathrm{YBa}_{2} \mathrm{Cu}_{4} \mathrm{O}_{8}$. Phys. Rev. Lett. 85, 4140-4143 (2000).

[42] Vojta, M., Zhang, Y. \& Sachdev, S. Quantum phase transitions in d-wave superconductors. Phys. Rev. Lett. 85, 4940-4943 (2000).

[43] Tsuei, C.C. \& Kirtley, J.R. Phase-sensitive evidence for d-wave pairing symmetry in electron-doped cuprate superconductors. Phys. Rev. Lett. 85, 182-185 (2000).

[44] Kokales, J.D. et al. Microwave electrodynamics of electron-doped cuprate superconductors. Phys. Rev. Lett. 85, 3696-3699 (2000).

[45] Prozorov, R. et al. Evidence for nodal quasiparticles in electron-doped cuprates from penetration depth measurements. Phys. Rev. Lett. 85, 3700-3702 (2000).

[46] Granath, M. et al. Nodal quasiparticles and coexisting orders in striped superconductors. Phys. Rev. Lett. 87, 167011-1-4 (2001).

[47] Belin, S. et al. Probing the upper critical field of $\kappa$-(BEDT-TTF $)_{2} \mathrm{Cu}(\mathrm{NCS})_{2}$. J. Supercon. 12, 497-500 (1999).

[48] Kleefisch, S. et al. Possible pseudogap behavior of electron doped high-temperature superconductors. Phys. Rev. B 63, 100507-100510 (2001).

[49] Osofsky, M.S. et al. Temperature dependence of the upper critical magnetic field in $\mathrm{BiSrCuO}$ and $\mathrm{NdCe}-$ $\mathrm{CuO}$. Proceedings of the 10th anniversary HTS workshop (World Scientific, Singapore, 1996), pp. 284-287.

[50] Bayot, V. et al. Evidence for weak localization in the thermal conductivity of a quasi-two-dimensional electron system. Phys. Rev. Lett. 65, 2579-2582 (1990).

[51] Berman, R. Thermal conduction in solids (Clarendon, Oxford 1976), p. 145

[52] Gutsmiedl, P., Probst. C. \& Andres, K. Low temperature calorimetry using an optical heating method. Cryogenics 31, 54-57 (1991). 